\title{
Does C3d assay predict positive cross-match: Potential additional biomarker for virtual cross-match.
}

Adarsh Babu 1,2,3, David Briggs ${ }^{3}$, Nithya Krishnan ${ }^{1}$, Dan Mitchell ${ }^{2}$ and Sunil Daga 1,2,3,

1. University Hospitals Coventry and Warwickshire. 2. University of Warwick. 3. NHSBT, Birmingham 4. Leeds Teaching Hospitals NHS Trust

\begin{tabular}{|c|c|c|c|}
\hline & Overall & CDC crossmatch & Flow crossmatch \\
\hline Sensitivity & $41.17 \%$ & $80 \%$ & $24.6 \%$ \\
\hline Specificity & $97.14 \%$ & $82.29 \%$ & $94.2 \%$ \\
\hline $\begin{array}{c}\text { Positive } \\
\text { predictive value } \\
\text { (PPV) }\end{array}$ & $97.24 \%$ & $54 \%$ & $88.21 \%$ \\
\hline $\begin{array}{c}\text { Negative } \\
\text { predictive value } \\
\text { (NPV) }\end{array}$ & $48 \%$ & $94 \%$ & $41.82 \%$ \\
\hline
\end{tabular}

Table 1: C3d assay has high specificity for predicting overall crossmatch

\section{Introduction}

HLA antigen matching and antibody crossmatch is performed prior to renal transplantation. It is well established that renal grafts have worse outcomes when transplanted across positive crossmatch. It was shown for the first time in 1969 that transplants across a positive crossmatch with complement activating antibodies had $80 \%$ graft failure within 3 months. IgG antibodies against donor pool human leucocyte antigens (HLA) are monitored in a potential recipient and crossmatch results are predicted on offer of potential kidney based on the HLA profile of the donor. Virtual crossmatch is widely performed in most centres and graft outcomes have shown similar to conventional crossmatch in matched donors. IgG anti HLA antibodies cause allograft damage by various immunological pathways. Currently $\operatorname{IgG}$ antibodies are studied to predict crossmatch. Recently studies are published that looking at C1q binding property of IgG antibodies and their role in virtual crossmatch. The studies have focussed if $\mathrm{Clq}$ binding property can predict flow or CDC crossmatch. The rationale being complement fixing/activating donor specific antibodies (DSA) (as measured by $\mathrm{C} 1 \mathrm{q} / \mathrm{C} 3 \mathrm{~d}$ binding) post transplantation is associated with poor graft outcome $\mathrm{e}^{4,5}$. Although, our study was looked at predictive value of complement activating antibodies for rejection and graft survival we correlated the findings to crossmatch results.

\section{Methods}

We analysed samples from 121 highly sensitised patients who had pre-transplant DSA and subsequently underwent direct transplantation between $2005-2015.86$ patients were crossmatch positive against their donors $25 \mathrm{CDC}$ and 61 Flow crossmatch (FC). Rest of the 35 patients only had DSA as detected by single antigen bead assay. C3d (Immucor) assay was performed at pre-transplant or preconditioning. Results were correlated with Flow cytometry and CDC crossmatches. Results were also correlated with early antibody mediated rejection (AMR) (rejection within the first 30 days) and allograft survival.

\section{Results}

C3d was positive in 37 cases pre-transplant. Of the 37 it was positive in 20/25 CDC positive cases, $15 / 61$ FC positive cases and in only 2/35 single antigen bead positive patients. From this results $\mathrm{C} 3 \mathrm{~d}$ positivity correlates well with crossmatch categorically. Sensitivity to predict CDC crossmatch is $80 \%$. Overall, the specificity is $97 \%$, in other words if the test is C3d is negative then we can be 97\% certain that CDC and Flow crossmatch will be negative.
Correlating the pre-transplant C3d DSA with outcomes, it did not correlate with predicting episodes of early rejection $(\mathrm{p}=1.00)$. In a Kaplan Meier survival analysis presence of IgG DSA that were C3d positive at pre-conditioning/pre-transplant correlated significantly with poor graft survival $(\mathrm{p}=0.001)$. In this cohort the graft outcomes of $\mathrm{C} 3 \mathrm{~d}$ positive cases were similar to outcomes predicted by CDC crossmatch.

\section{Discussion}

In this study although, was specifically designed to study C3d and correlation and crossmatch it has important message where it complement activation assays correlated with crossmatch resuls. Recent publications also support this finding. In our study we have shown that course of renal allograft that are $\mathrm{C} 3 \mathrm{~d}$ positive behaves similar to $\mathrm{CDC}$ positive crossmatch group. We cannot conclude from this study if complement activation assays can definitely replace crossmatch. Further larger studies of similar correlation to crossmatch and outcomes are necessary. Currently multicentre retrospective analysis is being conducted and the results from that will definitely provide additional robust information.

\section{References}

1. Arreola-Guerra, J. M., et al. (2016). "C1Q Assay Results in Complement-Dependent Cytotoxicity Crossmatch Negative Renal Transplant Candidates with Donor-Specific Antibodies: High Specificity but Low Sensitivity When Predicting Flow Crossmatch." J Transplant 2016: 2106028.

2. Juhl, D., et al. (2017). "Assignment of C1q-binding HLA antibodies as unacceptable HLA antigens avoids positive CDC-crossmatches prior to transplantation of deceased donor organs." Transpl Immunol.

3. Schaub, S., et al. (2014). "Determinants of C1q Binding in the Single Antigen Bead Assay." Transplantation 98(4): 387-393.

4. Loupy, A., et al. (2013). "Complement-Binding Anti-HLA Antibodies and Kidney-Allograft Survival." New England Journal of Medicine 369(13): 1215-1226.

5. Sicard, A., et al. (2015). "Detection of C3d-Binding Donor-Specific Anti-HLA Antibodies at Diagnosis of Humoral Rejection Predicts Renal Graft Loss." Journal of the American Society of Nephrology 26(2): 457-467. 